\title{
Progressive Muscle Relaxation therapy in Anxiety: A Neurophysiological Study
}

\author{
L. Ranjita ${ }^{1}$, Sarada.N ${ }^{2}$ \\ ${ }^{\text {I}}$ (Department of Physiology, Regional Institute of Medical Sciences, India) \\ ${ }^{2}$ (Department of Physiology, Regional Institute of Medical Sciences, India)
}

\begin{abstract}
Anxiety is a normal reaction to stress and represent a common human emotion. But when anxiety becomes an excessive, irrational dread of everyday situations, it becomes a disabling disorder. A reaction opposite to stress is relaxation. Relaxation techniques include behavioural therapeutic approaches that differ widely in philosophy, methodology, and practice. The objectives of the study were to analyse the EEG changes associated with Modified Jacobson's Progressive Muscle Relaxation Therapy (JPMRT) in stress management, to analyse the hemodynamic changes with relaxation and to validate the effectiveness of Modified JPMRT. Thirty-five patients diagnosed with Generalised Anxiety Disorder (GAD) as defined by the Standardised diagnostic criteria of DSM-IV-TR of American Psychiatric Association were subjected to Modified JPMRT for a total of ten sessions everyday by an experienced Clinical Psychologist. EEG, PR and BP were recorded and Hamilton rating scale for anxiety (HRSA) was taken before the beginning and at the end of therapy. Statistical analysis was done using ANOVA- single factor. Results showed a significant reduction in EEG frequency, PR, SBP and subjective HRSA scale. However, no significant reduction in DBP was observed. The present study confirms and extends the finding of efficacy of Modified JPMRT on GAD. It could prove as a valid treatment option for anxiety and depression related disorders.
\end{abstract}

Keywords: Anxiety, DBP, EEG, HRSA, JPMRT, PR, SBP

\section{Introduction}

Psychosocial stress is a pervasive aspect of modern life for most individuals, and there is evidence that such stress is associated with both increased morbidity and increased utilization of health care $[1,2]$. Anxiety is a normal reaction to stress and represent a common human emotion. But when anxiety becomes an excessive, irrational dread of everyday situations, it becomes a disabling disorder. A reaction opposite to stress is relaxation.

Relaxation techniques include behavioural therapeutic approaches that differ widely in philosophy, methodology, and practice. Relaxation strategies have been used for centuries as integral components of major philosophical, theological and therapeutic traditions. It is such a ubiquitous component of behaviour therapy that it has been referred to as "behaviours aspirin". In general, relaxation techniques refer to any strategies aimed at reducing arousal. One such method is Modified Jacobson's Progressive Muscle Relaxation Therapy (JPMRT) which stems primarily from the work of Edmund Jacobson in early 1920s. PMR originated from the theory that a psychobiological state called neuromuscular hypertension is the basis for a variety of negative emotional states and psychosomatic diseases. Jacobson asserted that relaxation of muscles would lead to relaxation of mind, "because an emotional state fails to exists in the presence of complete relaxation of peripheral parts involved" [3].

A broad understanding of the etiology of anxiety problems includes a multiplicity of factors, such as biological, psychological, and social determinants, which are mediated by a range of risk and protective factors [4]. The old debate over the primacy of these factors, overall biological or psychological, is gradually being replaced by a pragmatic model considering all the relative contributions. Clinical trials have shown that anxiolytic drugs alone have limited long-term efficacy [5]. Moreover, they often have adverse side effects including dependency, drowsiness, impaired cognition and memory and sexual dysfunction $[6,7,8]$. Consequently, clinical community has begun to consider alternative old and new approaches targeting anxiety problems and to examine the merits of combined and tailored somatic and psychological treatments.

Different conscious states are accompanied by different neuro-physiological states and the brain electrical activities measured would reflect this changes. This assumption is based on the association between particular electroencephalograph (EEG) patterns of cortical activity and specific states of cognitive or psychological function. Hence, the present study aims to explore the effectiveness of JPMRT by analysing the changes in EEG wave pattern along with associated changes in hemodynamic variables i.e. Pulse Rate (PR) and Blood Pressure (BP). 


\section{Material and methods}

The study was conducted in the Departments of Physiology, Psychiatry and Clinical Psychology of Regional Institute of Medical Sciences (RIMS), India, during the period from January 2012 to August 2013 after getting approval from the Institute's ethical committee. 35 (thirty-five) patients diagnosed with Generalised Anxiety Disorder (GAD) as defined by the Standardised diagnostic criteria of DSM-IV-TR of American Psychiatric Association was recruited from outpatient department, Psychiatry by a Psychiatry consultant. Inclusion criteria:

- Individuals between 18-50 years of age.

- Both males and females were taken for the study.

Exclusion criteria:

- Seriously ill patients.

- Patients with past or present clinical evidence of drug/substance abuse or general medical conditions (like hyper- or hypo-thyroidism, epilepsy, asthma etc.), head injury, major surgical operations etc..

Parameters studied:

i. $\quad$ EEG recording

ii. Hemodynamic variables viz. pulse rate (PR), Blood pressure (BP)

iii. Subjective Hamilton Scoring scale for anxiety

Prior to test, all subjects underwent a detail history taking, general physical examination and systemic examination. Informed consent was obtained from all participants. The subjects were explained about the nature and purpose of the study. Then, they were subjected to Modified Jacobson Progressive Muscle Relaxation Therapy (JPMRT) for a total of 10 (ten) sessions everyday by an experienced Clinical Psychologist in the Department of Clinical Psychology, RIMS. Participants learnt the techniques as well as their fundamental principles during the first training session. They were encouraged to practise the techniques at home regularly. In order to ensure that the participants practised regularly at home, a log record of daily practice frequency and duration was maintained.

Progressive muscle relaxation is a stress management technique developed by Jacobson, which is a widely practised relaxation method and has been reported to be effective in alleviating anxiety and mood problems. Participants were taught according to the standard procedure of the technique: beginning with some deep breaths with closed eyes, followed by alternately tensing and relaxing groups of muscles in a prescribed sequence from the head down along the chest, the hands, and eventually the feet with the specific intention of developing an acute awareness of the difference. Participants were to inhale when tensing the muscles, exhale when relaxing, and feel the bodily changes throughout the tensing/relaxing cycle.

The subjects were made to rest and sit quietly for about 5 minutes duration before any recording was done. Baseline recording of EEG was made prior to Modified JPMRT and at the end of session. EEG recording was done using a single channel Physiograph Recorder, Students' Physiograph model from Recorders and Medicare Systems (P) Ltd., Chandigarh, India. The signal is fed into the amplifier directly by using suitable electrodes. 3 (three) flat electrodes are positioned at (1) mid-forehead (2) mid-occipital and (3) left mastoid, after cleaning the scalp with acetone and immersing the electrodes in colloidal paste. The electrode on the left mastoid is relatively inactive electrically and it serves as ground electrode. Impedence was kept at less than 10kohms for each electrode. Input switch was maintained at time constant of $0.3 \mathrm{~s}$, sensitivity at $0.1 \mathrm{mV}$ with chart speed at $25 \mathrm{~mm} / \mathrm{s}$. The sampling frequency was $250 \mathrm{~Hz}$ with cut off frequency at $50 \mathrm{~Hz}$. EEG recording was made before and after relaxation therapy. The data was stored on chart paper for analysis.

Simultaneously, hemodynamic variables i.e., pulse rate and blood pressure were measured before and after relaxation therapy in sitting position after letting the subjects to rest for 5 minutes.

The subjective score for anxiety was made by interviewing the subjects using Hamilton Rating Scale for Anxiety before and after relaxation therapy.

Statistical techniques like mean, standard deviation, one way- analysis of variance (ANOVA), Pearson's correlation coefficient and Chi-square test were used whenever found suitable and necessary for analysing the data using Microsoft Office Excel 2003. Accordingly, interpretations are made. A probability value of $p$-value $<0.05$ was taken as statistically significant.

\section{Result}

The data collected from the subjects who underwent Modified Jacobson Progressive Muscle Relaxation Therapy is organized and presented under the following headings:

Section I: Demographic characteristics.

Section II: To test the effectiveness of therapy.

a) Effect of therapy on stress. 
b) Effect of therapy on physiological parameters.

Table 1: Demographic character of the patients

\begin{tabular}{|c|c|c|}
\hline \multirow{2}{*}{ Age (Years) } & $18-28$ & 8 \\
& $29-39$ & 14 \\
& $40-50$ & 13 \\
\hline \multirow{2}{*}{ Gender } & Male & 20 \\
& Female & 15 \\
\hline \multirow{2}{*}{ Religion } & Hindu & 26 \\
& Muslim & 5 \\
\hline
\end{tabular}

Maximum patients belong to Hindu males with age group of 29-39 years.

Table 2: Frequency and percentage distribution of EEG waves in GAD subjects $(\mathbf{N}=35)$

\begin{tabular}{|l|c|c|c|c|}
\hline \multirow{2}{*}{$\begin{array}{c}\text { EEG - wave } \\
\text { (Anxiety) }\end{array}$} & \multicolumn{2}{|c|}{ Pre - JPMRT } & \multicolumn{2}{c|}{ Post - JPMRT } \\
\cline { 2 - 5 } & $N=35$ & $\%$ & $N=35$ & $\%$ \\
$\beta$ - wave & 0 & $0 \%$ & 0 & $40 \%$ \\
$\gamma$ - wave & 1 & $3 \%$ & 14 & $60 \%$ \\
$\delta$ - wave & 34 & $97 \%$ & 21 & $0 \%$ \\
\hline
\end{tabular}

Table 3: Mean, Standard deviation, F-value and P-value of physiological parameter (PR, SBP, DBP, EEG) in GAD subjects $\mathbf{N}=\mathbf{3 5}$

\begin{tabular}{|l|c|c|c|c|}
\hline \multirow{2}{*}{ Parameters $(N=35)$} & Pre - JPMRT & Post - JPMRT & \multirow{2}{*}{ F-value } & \multirow{2}{*}{ P-value } \\
\cline { 2 - 5 } & Mean \pm SD & Mean \pm SD & 34.95 & 0 \\
\hline Pulse Rate (beats/min) & $80.57 \pm 6.43$ & $73.14 \pm 3.73$ & 10.34 & 0.002 \\
\hline SBP $(\mathrm{mmHg})$ & $130.69 \pm 8.58$ & $124.17 \pm 8.36$ & 3.55 & 0.064 \\
\hline DBP $(\mathrm{mmHg})$ & $83.71 \pm 5.80$ & $80.91 \pm 6.60$ & 70.16 & 0 \\
\hline EEG $(\mathrm{Hz})$ & $35.86 \pm 2.43$ & $31.2 \pm 2.22$ & 67.2 & 0.00 \\
\hline Anxiety score & $9.54 \pm 1.87$ & $6.54 \pm 1.09$ & &
\end{tabular}

$P$-value $<0.05$ is considered significant

Comparison of base-line Pulse rate, SBP and EEG frequency at pre- and post- Progressive muscle relaxation therapy showed a trend of significant reduction, however, no significant change was seen in case of DBP before and after Progressive muscle relaxation therapy.

Before the beginning of Progressive muscle relaxation therapy, 97\% of the subjects showed gamma wave frequency. After the end of therapy, $60 \%$ showed gamma wave and $40 \%$ showed beta wave. Alpha wave, which is indicative of relaxation, was not recorded, though, there was significant reduction in EEG frequency at the end of therapy.

\section{Discussion}

The present study evaluates the impact of Modified JPMRT on stress. The ability to generate alpha brainwaves has been associated with the self regulation of stress (Wacker 1996). Previous studies specifically linked anxiety and relaxation with EEG recordings (Isotani et al 2001) and found that an increase in alpha frequencies in the frontal scalp area is an indication of positive relaxation training effects of audiovisual stimulation [9]. Although alpha changes are tightly linked to anxiety changes, this is only evident in highanxiety subjects as discovered by Hardt and Kamiya [10] . Their study showed that some people with high levels of anxiety have low alpha waves, and EEG alpha increase is beneficial only for patients who exhibit this low amplitude alpha. EEG alpha levels had been thought to increase during relaxation and decrease during stress. However in the present study, no alpha wave was observed, though there was significant change from $\gamma$ wave in pre- relaxation therapy to $\beta$-wave at post- relaxation therapy. Alpha level was also shown not to be related to muscular relaxation as it was maintained even during vigorous muscle tension. ${ }^{11}$ Failure to find alpha waves could be because either the patients may not actually have learned the primary skills of relaxation or due to short duration of JPMRT. Moreover, the patients were all beginners with no previous experience of the therapy.

However, PR and SBP showed a significant decline at the end of JPMRT. This finding is consistent with the works of Neves [12] whose work concluded that patients who participated in relaxation therapy improved their 
cardiovascular risk factors profile. This findings is also consistent with findings of van Dixhoorn et al. and Collins and Rice $[13,14]$. Studies by Blumenthal JA, Collins and Kanji.N also showed a reduction in PR but no change in SBP and DBP $[15,16]$.

Subjective Hamilton rating for anxiety score was significantly reduced at the end of JPMRT compared to preJPMRT. This is consistent with the finding of Hawkins [17], Conrad and Roth [3] and Chen [18].

The relationship of stress management and relaxation therapy is more complex and depends upon definition. Traditionally, stress management programmes are based upon relaxation training, and full relaxation training was seen as a form of stress management [9], because it helps individuals to deal with stress and tension. The classical relaxation methods aim to reduce the strain in the individual, which was seen to have accumulated in the course of time and exposures to stressors. In progressive muscle relaxation it is neuromuscular hypertension or elevated muscle tension [19].

This study demonstrates that relaxation techniques may offer such an easy to learn healthy alternative for subjective stress and state anxiety regulation.

The present results should be viewed in the light of several methodological limitations. Our study did not examine potential physiological and/or psychological mechanisms that are responsible for the reduced state anxiety, psychological distress and improved subjective well-being. It is not known whether the PMR may have exerted its effects merely as a diversion or as welcomed evidence to patients that their psychosocial needs were being acknowledged. It is difficult to distinguish the effects of JPMRT on EEG frequency from confounding conditions like drowsiness, medications, caffeinated drinks, changes in emotional state/arousal, artefact from eye movements, time of day, and state of alertness.

\section{Conclusion}

The present study confirms and extends the finding of efficacy of Modified Jacobson Progressive Muscle Relaxation Therapy (JPMRT) on GAD. The significant reduction in anxiety scores could lead to a big improvement in the morbidity and mortality rate as the result indicate that relaxation therapy is effective in any kind of participants, male or female, young or old affected with psychological disorders. Besides, it is easy to learn and practice, does not need any equipment to facilitate the training and is cost effective. It could prove as a valid treatment option for anxiety and depression related disorders.

\section{References}

[1] Levi L, Society, Stress and Disease: The Psychosocial Environment and Psychosomatic Disease (London: Oxford University Press, 1971).

[2] Dohrenwend BS, Dohrenwend DP, Stressful Life Events: Their Nature and Effects (New York: John Wiley, 1974) 73-86.

[3] Conrad A, Roth WT, Muscle relaxation therapy for anxiety disorders:It works but how?, Journal of anxiety disorder, 21, 2007, 243264.

[4] Somers JM, Goldner EM, Waraich P, Hsu L, Prevalence and incidence studies of anxiety disorders: a systematic review of the literature, Can J Psychiatry, 51(2), 2006, 100-113.

[5] Youngstedt SD, Kripke DF, Does bright light have anxiolytic effects? - an open trial, BMC Psychiatry ,7(1), $2007,62$.

[6] Buffett-Jerrott SE, Stewart SH, Cognitive and sedative effects of benzodiazepine use. Curr Pharm Des, 8(1), 2002, 45-58.

[7] Struzik L, Vermani M, Coonerty-Femiano A, Katzman MA, Treatments for generalized anxiety disorder. Expert Rev Neurother, 4(2), 2004, 285-294.

[8] Segraves RT, Sexual side-effects of psychiatric drugs. Int J Psychiatry Med, 18(3), 1988, $243-252$.

[9] Sterman MB, Howe RD, Macdonald LR, Facilitation of spindle burst sleep by conditioning of electroencephalographic activity while awake, Science, 167, 1970, 1146-1148.

[10] Hardt JV, Kamiya J, Anxiety change through electroencephalographic alpha feedback seen only in high anxiety subjects, Science, 201, 1970, 79-81.

[11] Brown BB, Awareness of EEG subjective activity relationships detected within a closed feedback system, Psychophysiology, 7, 1971, 451-464.

[12] Neves A, Alves AJ, Ribeiro F, Gomes JL, Oliveira J, The effect of cardiac rehabilitation with relaxation therapy on psychological, hemodynamic, and hospital admission outcome variables, Journal of Cardiopulmonary Rehabilitation and Prevention, 29, 2009, 304-309.

[13] Van Dixhoorn JJ, Duivenvoorden HJ, Effect of relaxation therapy on cardiac events after myocardial infarction: a 5-year follow-up study, J Cardiopulm Rehabil, 19, 1999, 178-185.

[14] Collins JA, Rice VH, Effects of relaxation intervention in phase II cardiac rehabilitation: replication and extension, Heart Lung, 26, 1997, 31-44.

[15] Blumenthal JA, Jiang W, Babyak MA, Krantz DS, Frid DJ, Coleman RE, et al, Stress management and exercise training in cardiac patients with myocardial ischemia. Effects on prognosis and evaluation of mechanisms, Arch Intern Med, 157, 1997, $2213-2223$.

[16] Kanji N, White AR, Ernst E, Autogenic training reduces anxiety after coronary angioplasty: a randomized clinical trial, Am Heart J , 147, 2004, E10, K1-K4.

[17] Hawkins RC, Doell SR, Lindseth P, Jeffers V, Skaggs S, Anxiety reduction in hospitalized schizophrenics through thermal biofeedback and relaxation training. Perceptual and Motor Skills, 51, 1980, 475-482.

[18] Chen WC, Chu H, Lu RB, Chou YH, Chen CH, Chang YC, O'Brien AP and Chou KR, Efficacy of progressive muscle relaxation training in reducing anxiety in patients with acute schizophrenia. Journal of Clinical Nursing, 18, 2009, $2187-2196$.

[19] McGuigan FJ, Progressive relaxation: origins, principles and clinical applications, in Lehrer PM, Woolfolk RL (Ed.), Principles and practice of stress management. (New York: Guilford Press, 1993) 17-52. 